\title{
Statistical Thermodynamic Analysis of Phosphorus \\ Solubility in Molten Iron*
}

\author{
By Nobumitsu SHOHOJI**
}

\begin{abstract}
Synopsis
Appreciable amount of phosphorus dissolves interstitially into mollen iron. A statistical thermodynamic analysis, based on a priori assumption of constant solute-solute interaction at a given temperature $T$, is applied to molten $\mathrm{FeP}_{x}$. The interaction energy, $E_{\mathrm{P}-\mathrm{Fe}}$, between $\mathrm{P}$ and $\mathrm{Fe}$ in molten $\mathrm{FeP}_{x}$ obtained by the analysis is about $-58 \mathrm{~kJ} \mathrm{~mol}^{-1}$ in the range of 1473 to $1773 \mathrm{~K}$. The interaction energy, $E_{\mathrm{P}-\mathrm{P}}$, between $\mathrm{P}$ atoms in molten $\mathrm{FeP}_{x}$ is estimated to be weak and altractive (varying from $\sim-1 \mathrm{~kJ} \mathrm{~mol}^{-1}$ at $1473 \mathrm{~K}$ to $\sim-2 \mathrm{~kJ} \mathrm{~mol}^{-1}$ at $1773 \mathrm{~K}$ ). Analysis is also made for some ternary molten $\mathrm{FeX}_{y} \mathrm{P}_{x}$, where $\mathrm{X}$ is another interstitial element $(\mathrm{X}=\mathrm{C}, \mathrm{B})$.
\end{abstract}

\section{Introduction}

Appreciable amount of phosphorus (P) dissolves interstitially into molten iron (Fe). Recently, Ban-ya et al. ${ }^{1)}$ measured the phosphorus partial pressure $p_{\mathrm{P}_{2}}$ in equilibrium with molten $\mathrm{FeP}_{x}(x<0.42)$ by the transportation method in the temperature range of 1473 to $1773 \mathrm{~K}$. Further, Ban-ya et al. ${ }^{2)}$ reported the equilibrium $p_{\mathrm{P}_{2}}-x$ relationships for the ternary $\mathrm{Fe}-\mathrm{X}-\mathrm{P}$ systems $(\mathrm{X}=\mathrm{B}, \mathrm{C}, \mathrm{Si}, \mathrm{Al})$ at $1673 \mathrm{~K}$. They analysed the experimental $p_{\mathrm{P}_{2}}-T_{-} x$ relationships on the basis of conventional thermodynamics. However, the experimental data in the composition range of $x>1 / 3$ were not used for their analysis, because a kink appeared around the composition of $\mathrm{Fe}_{3} \mathrm{P}$ in the plot of the apparent equilibrium constant $\log K_{1}^{\prime}(Z)$ against $Y_{\mathrm{P}}$ ( $x$ in the present notation) ( $c f ., \mathrm{Eq} .(19)$ and Fig. 5 in Ref. 1)). According to the results of the recent statistical thermodynamic analyses of several interstitial solid solutions $\mathrm{MX}_{x},{ }^{3-12)}$ this type of kink or discontinuity of the plots may be avoided by properly choosing the number $\theta$ of the available interstices for occupation by the interstitial atom $\mathrm{X}$ per host metal atom $M$. The choice of the value of parameter $\theta$ may be made so that the linear

$$
\underline{\mathrm{A}} \equiv R T \ln \left(\frac{\theta-x}{x} p_{\mathrm{x}_{2}}^{1 / 2}\right) \text { vs. } x
$$

relationship yields at a given temperature. This is to satisfy a priori assumption of constant solute-solute interaction at a given temperature.

The purpose of the present study is to analyse the available $p_{\mathrm{P}_{2}}-T-x$ relationships reported by Ban-ya et al. ${ }^{1,2)}$ in terms of statistical thermodynamics, rather than the conventional thermodynamics, on the basis of a priori assumption of constant $\mathrm{P}-\mathrm{P}$ interaction at a given temperature in molten $\mathrm{FeP}_{x}$ and $\mathrm{FeX}_{y} \mathrm{P}_{x}(\mathrm{X}=$ $\mathrm{B}, \mathrm{C})$.

\section{Method of Analysis}

As has been employed commonly in the recent analyses $^{3-12)}$ for interstitial solid solutions, the fundamental equation:

$$
R T \ln \left(\frac{\theta-x}{x} p_{\mathrm{P}_{2}}^{1 / 2}\right)=g+\beta x E_{\mathrm{P}^{2}-\mathrm{P}}
$$

is used for the present analysis of molten $\mathrm{FeP}_{x}$, where, $R$ refers to the gas constant and $p_{\mathrm{P}_{2}}$ the phosphorus partial pressure (in atm). In molten $\mathrm{FeP}_{x}, \mathrm{Fe}$ atoms may be assumed to be in the close packed arrangement (fcc; face centred cubic). ${ }^{1,2}$ Then, the parameter $\beta$ in Eq. (1) is equal to 12 and $x$-independent factor $g$ may be expressed as

$$
\begin{aligned}
& g=K-\mu_{\mathrm{P}}^{\circ}, \quad \ldots \ldots \ldots \ldots \\
& K=6 E_{\mathrm{P}-\mathrm{Fe}}-R T \ln f_{\mathrm{P}}
\end{aligned}
$$

where, $f_{\mathrm{P}}$ : the atomic partition function of $\mathrm{P}$ in molten $\mathrm{FeP}_{x}$

$\mu_{\mathrm{P}}^{\circ}$ : the chemical potential of the gaseous phosphorus with the reference state being taken as the gaseous phosphorus atom at rest.

The expression for $\mu_{\mathrm{P}}^{\circ}$ may be given as

$$
\begin{aligned}
& \mu_{\mathrm{P}}^{\circ}=R T C(T)-D_{\mathrm{P}_{2}} / 2 \\
& C(T)=-\frac{1}{2} \ln \left(\frac{\left(4 \pi m_{\mathrm{P}}\right)^{3 / 2} k^{5 / 2}}{2 h^{3}} \frac{T^{7 / 2}}{\theta_{r}}\right) \\
& +\frac{\theta_{v}}{4 T}+\frac{1}{2} \ln \left(1-\exp \left(-\theta_{v} / T\right)\right)
\end{aligned}
$$

where, $h$ : the Planck constant

$k$ : the Boltzmann constant

$m_{\mathrm{P}}:$ the mass of $\mathrm{P}$ atom

$\theta_{v}(=1123 \mathrm{~K}), \theta_{r}(=0.436 \mathrm{~K})$ : the characteristic temperatures for vibration and rotation, respectively, of $\mathrm{P}_{2}$ molecule

$D_{\mathrm{P}_{2}}$ : the dissociation energy per mole of $\mathrm{P}_{\mathbf{2}}$ molecule (e.g., Ref. 13)).

In the following, a brief explanation will be given to the reason why a priori assumption of constant solute-solute interaction at a given temperature is employed. It has been a common practice in the analysis of interstitial solutions (e.g., Refs. 8, 9, 13-19)) to take the value of parameter $\theta$ to be equal to the total number $\theta_{0}$ of the relevant interstices per metal atom. Then, the estimated interaction energy, $E_{i j}$, between component atoms, $i$ and $j$, in $\mathrm{MX}_{x}$ were found to

\footnotetext{
* Manuscript received on June 25, 1985; accepted in the final form on October 11, 1985. (C) 1986 ISIJ

** Departamento de Metalurgia e Metalomecânica, Laboratório Nacional de Engenharia e Tecnologia Industrial, 1699 Lisboa Codex, Portugal.
} 
hold constant. These results had been appreciated as rational and acceptable. However, in the analysis of hypostoichiometric chromium subnitride $\mathrm{Cr}_{2} \mathrm{~N}$, an apparent anomality was observed ${ }^{3)}$; i.e., with $\theta=1$ (which corresponds to the random distribution of $\mathrm{N}$ atoms over all the available O-sites (octahedral interstices) in hcp (hexagonal close packed) Cr-lattice), the value of $E_{\mathrm{N}-\mathrm{N}}$ varies with $\mathrm{N}$-content at a given temperature. This unexpected variation of $E_{N-N}$ with $\mathrm{N}$-concentration was found to be avoided by the choice of $\theta=0.5 .^{3)} \quad$ This value of $\theta$ suggests that only half of the total O-sites would provide as available sites for the occupation by $\mathrm{N}$ atoms. This model appears to be compatible with the X-ray diffraction observation $^{20}$ ) showing the ordered distribution of $\mathrm{N}$ atoms over the O-sites in hcp Cr-lattice. Further analyses of miscellaneous interstitial solutions on the basis of the assumption of constant $E_{\mathrm{X}-\mathrm{x}}$ at a given temperature led to the following conclusions ${ }^{4-10)}$ :

(1) Similar atomic partition function $f_{\mathrm{X}}$ as well as the similar interaction energy term $E_{\mathrm{X}-\mathrm{M}}$ is derived for the metals belonging to the same group in the periodic table, which appears to be realistic, judging from the similarity of the electronic structure of the metals of same group.

(2) The value of parameter $\theta$ is estimated to be close to the maximum solubility limit $x^{\prime}$ of $\mathrm{MX}_{x^{\prime}}$ $\left(\theta>x^{\prime}\right)$.

These results seem to support the employment of $a$ priori assumption of constant $E_{\mathrm{X}-\mathrm{X}}$ at a given temperature. Without this assumption, the statistical thermodynamic analysis of interstitial solution may become too much arbitrary; e.g., $E_{\mathrm{H}-\mathrm{H}}$ in $\mathrm{YH}_{x}$ may be estimated to be either positive (repulsive) or negative (attractive) depending on the choice of $\theta .{ }^{21}$ ) There is no justification to choose some value of $\theta$ based simply on the crystal structure being considered.

\section{Results and Discussion}

1. Molten $\mathrm{FeP}_{x}$

In order to determine the value of the parameter $\theta$ for $\mathrm{FeP}_{x}, \underline{\mathrm{A}} v s . x$ plots are prepared using the experimental $p_{\mathrm{P}_{2}}-x$ relationship at $1673 \mathrm{~K}^{1)}$ with various choices of 0 -values, as shown in Fig. 1. As is clear from Eq. (1), the slope of $\underline{\mathrm{A}} v s . x$ plots is proportional to $E_{\mathrm{P}-\mathrm{P}}$. Judging from the results summarized in Fig. 1, the choice of $\theta=0.5$ appears to satisfy $a$ priori assumption of constant $E_{\mathrm{P}-\mathrm{P}}$ at a given temperature. Thus, $\theta=0.5$ is employed for the analysis of molten $\mathrm{FeP}_{x}$. This choice of $\theta$-value may imply that half the number of total O-sites in "molten fcc lattice" of $\mathrm{Fe}$ is subjected to the occupation by $\mathrm{P}$ atoms alternately.

Figure 2 shows the plots of $\underline{\mathrm{A}}$ vs. $x$ for molten $\mathrm{FeP}_{x}$ with $\theta=0.5$. From these plots, the value of $\beta E_{\mathrm{P}-\mathrm{P}}$ and $K$ may be derived as a function of temperature (cf., Fig. 3). Interaction between $\mathrm{P}$ atoms in molten $\mathrm{FeP}_{x}$ appears to be attractive and slightly dependent on temperature (varying from $\sim-1 \mathrm{~kJ} \mathrm{~mol}^{-1}$ at 1473 $\mathrm{K}$ to $\sim-2 \mathrm{~kJ} \mathrm{~mol}^{-1}$ at $1773 \mathrm{~K}$ ). From the $K-T$ relationship summarized in Fig. 3, the following parameters may be estimated, when $E_{\mathrm{P}-\mathrm{re}}$ and $f_{\mathrm{P}}$ are assumed to be independent of temperature:

$$
\begin{aligned}
& 6 E_{\mathrm{P}-\mathrm{Fe}_{\mathrm{e}}}=-347.1 \mathrm{~kJ} \mathrm{~mol}^{-1} \\
& R \ln f_{\mathrm{P}}=91.5 \mathrm{~J} \mathrm{~K}^{-1} \mathrm{~mol}^{-1}
\end{aligned}
$$

The atomic partition function $f_{\mathrm{P}}$ is in fact a function which is dependent on temperature. However, as discussed in the earlier work ( $c f$. , Appendix in Ref. 8)), this approximation would not cause very serious error in the estimation of $E_{\mathrm{P}-\mathrm{Fe}}$ over a temperature range of a few hundred Kelvin.

\section{Molten $\mathrm{FeX}_{y} \mathrm{P}_{x}(\mathrm{X}=\mathrm{B}, \mathrm{C})$}

Ban-ya et al. ${ }^{2)}$ reported that $\mathrm{Si}$ and $\mathrm{Al}$ form substitutional solutions with molten Fe but that B and $\mathrm{C}$ dissolve interstitially into molten $\mathrm{Fe}$. In the following, analysis of the $p_{\mathrm{P}_{2}}-x$ relationships for $\mathrm{FeX}_{y} \mathrm{P}_{x}$ $(\mathrm{X}=\mathrm{B}, \mathrm{C})$ at $\left.1673 \mathrm{~K}^{2}\right)$ is given.

For the analysis of $\mathrm{FeX}_{y} \mathrm{P}_{x}$, a modified version of Eq. (1) must be used; i.e.,

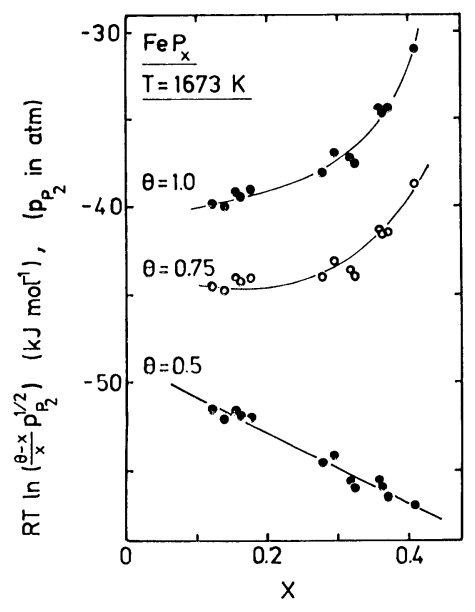

Fig. 1. Effect of the choice of parameter $\theta$ on $R T \ln \left(\frac{\theta-x}{x} p_{\mathrm{P}_{2}}^{1 / 2}\right)$ vs. $x$ plots for molten $\mathrm{FeP}_{x}$ at $1673 \mathrm{~K}$.

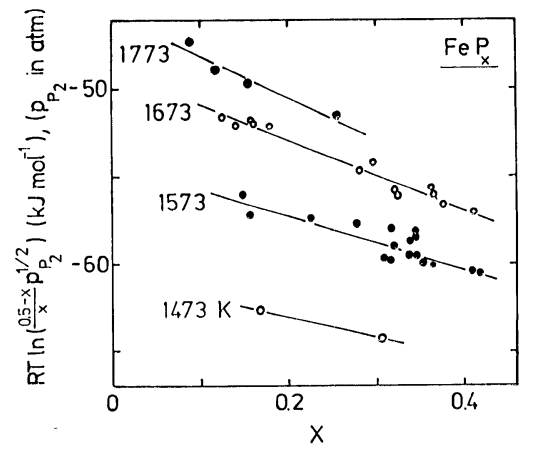

Fig. 2. $R T \ln \left(\frac{0.5-x}{x} p_{\mathrm{P}_{2}}^{1 / 2}\right) v s . x$ plots for molten $\mathrm{FeP}_{x}$.

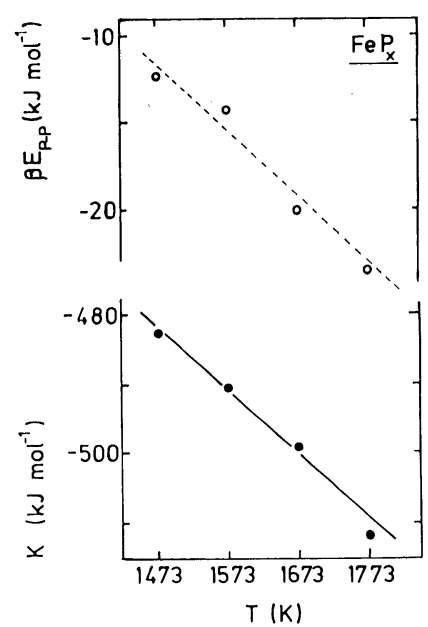

Fig. 3. Values of $\beta E_{\mathrm{P}-\mathrm{P}}$ and $\kappa$ for molten $\mathrm{FeP}_{x}$ as a function of $T$. 


$$
\begin{aligned}
R T \ln \left(\frac{0-x}{x} p_{\mathrm{P}_{2}}^{1 / 2}\right)= & \left(6 E_{\mathrm{P}-\mathrm{Fe}}+12 y E_{\mathrm{X}-\mathrm{P}}\right. \\
& \left.-R T \ln f_{\mathrm{P}}-\mu_{\mathrm{P}}^{\circ}\right)+12 x E_{\mathrm{P}-\mathrm{P}}
\end{aligned}
$$

This modification is resulted from the alteration of the lattice energy $E$ of $\mathrm{FeX}_{y} \mathrm{P}_{x}$ from that of $\mathrm{FeP}_{x}$ :

$$
\begin{aligned}
E\left(\mathrm{FeX}_{y} \mathrm{P}_{x}\right)= & 6 n_{\mathrm{Fe}} E_{\mathrm{Fe}-\mathrm{Fe}}+6 n_{\mathrm{X}} E_{\mathrm{X}-\mathrm{Fe}}+6 n_{\mathrm{P}} E_{\mathrm{P}-\mathrm{Fe}} \\
& +6 \frac{n_{\mathrm{X}}^{2}}{n_{\mathrm{Fe}}} E_{\mathrm{X}-\mathrm{X}}+12 \frac{n_{\mathrm{X}} n_{\mathrm{P}}}{n_{\mathrm{Fe}}} E_{\mathrm{X}-\mathrm{P}} \\
& +6 \frac{n_{\mathrm{P}}^{2}}{n_{\mathrm{re}}} E_{\mathrm{P}-\mathrm{P}}, \ldots \ldots \ldots \ldots \ldots \ldots \ldots \ldots \ldots \ldots \ldots \ldots \ldots \ldots
\end{aligned}
$$

where, $n_{i}(i=\mathrm{Fe}, \mathrm{X}, \mathrm{P})$ refers to the number of component atom $i$ in molten $\mathrm{FeX}_{y} \mathrm{P}_{x}$. Interstitial elements, $\mathrm{B}$ and $\mathrm{C}$, as well as $\mathrm{P}$ are supposed to occupy the $\mathrm{O}$-sites in molten Fe-lattice.

The number $W$ of configuration of interstitial atoms in molten $\mathrm{FeX}_{y} \mathrm{P}_{x}$ may be given as

$$
W\left(\mathrm{FeX}_{y} \mathrm{P}_{x}\right)=\frac{\left(\theta^{\prime} n_{\mathrm{Fe}}\right) !}{\left(\theta^{\prime} n_{\mathrm{re}}-n_{\mathrm{X}}\right) ! n_{\mathrm{X}} !} \frac{\left(\theta n_{\mathrm{Fe}}\right) !}{\left(\theta n_{\mathrm{Fe}}-n_{\mathrm{P}}\right) ! n_{\mathrm{P}} !}
$$

instead of

$$
W\left(\mathrm{FeP}_{x}\right)=\frac{\left(\theta n_{\mathrm{Fe}}\right) !}{\left(\theta n_{\mathrm{Fe}}-n_{\mathrm{P}}\right) ! n_{\mathrm{P}} !}
$$

where, the parameter $\theta^{\prime}$ refers to the number of available interstices for $\mathrm{X}$ atoms per $\mathrm{Fe}$ atom. This alteration of $W\left(\mathrm{FeX}_{y} \mathrm{P}_{x}\right)$ from $W\left(\mathrm{FeP}_{x}\right)$ does not affect the apparent functional form of the left-hand side of the equilibrium formula ( $c f$. Eqs. (1) and (8)). However the parameter $\theta$ in $\mathrm{FeX}_{y} \mathrm{P}_{x}$ does not possess the same value as that in $\mathrm{FeP}_{x}$, but may deviate from 0.5 as a function of $y=n_{\mathrm{X}} / n_{\mathrm{re}}$ due to the blockage of the interstitial sites by $\mathrm{X}$ atoms.

If it is assumed that $E_{\mathrm{X}-\mathrm{P}}$ is negligible and that $E_{\mathrm{P}-\mathrm{P}}, E_{\mathrm{P}-\mathrm{re}}$ and $f_{\mathrm{P}}$ in $\mathrm{FeX}_{y} \mathrm{P}_{x}$ are not affected by the addition of $\mathrm{X}$ and maintain approximately the same value as in $\mathrm{FeP}_{x}$, Eq. (8) for $\mathrm{FeX}_{y} \mathrm{P}_{x}$ may be reduced to the same form as Eq. (1) with only the value of the parameter $\theta$ deviating from 0.5 . In this approximation, the effect of the addition of interstitial element $\mathrm{X}$ is simply reduced to the blockage of the available interstices.

Equilibrium relation for $\mathrm{FeP}_{x}$ at $1673 \mathrm{~K}$ may be expressed as follows ( $c f$., Fig. 2):

$$
\ln \left(\frac{\theta-x}{x} p_{\mathrm{P}_{2}}^{1 / 2}\right)=-3.514-1.44 x
$$

with $\theta=0.5$. From the above mentioned approximation for $\mathrm{FeX}_{y} \mathrm{P}_{x}$, the value of $\theta$ as a function of $y$ may be estimated from the following equation:

$$
\theta=x\left(1+\exp \left(-3.514-1.44 x-\frac{1}{2} \ln p_{\mathrm{P}_{2}}\right)\right)
$$

The results of this estimation for $\mathrm{X}=\mathrm{B}$ and $\mathrm{G}$ are summarized in Fig. 4. It appears that $\mathrm{C}$ blocks the interstices more extensively than $B$ per fraction of addition, provided that the approximation is valid.

The analysis for the substitutional addition $\mathrm{Fe}_{1-z}$ $\left.\mathrm{X}_{z} \mathrm{P}_{x}(\mathrm{X}=\mathrm{Si}, \mathrm{Al})^{2}\right)$ was not made, since the alteration of the electronic structure of the host metal lattice $\mathrm{Fe}_{1-z} \mathrm{X}_{z}$ from pure $\mathrm{Fe}$ may cause quite significant changes in $E_{\mathrm{P}-\mathrm{P}}$ and the atomic partition function $f_{\mathrm{P}}$ and, further, even the explicit functional form for $K$ may be altered to

$$
K\left(\mathrm{Fe}_{1-z} \mathrm{X}_{z} \mathrm{P}_{x}\right)=6(1-z) E_{\mathrm{P}-\mathrm{Fe}}+6 z E_{\mathrm{P}-\mathrm{X}}-R T \ln f_{\mathrm{P}}
$$

in place of Eq. (3).

\section{Re-construction of $p_{\mathrm{P}_{2}}-T-x$ Relationships}

Using the parameters estimated from this analysis, $p_{\mathrm{P}_{2}}-T-x$ relationships may be re-constructed for $\mathrm{FeP}_{x}$ and for $\mathrm{FeX}_{y} \mathrm{P}_{x}$. Equations may be as follows:

$$
\begin{aligned}
\log _{10} p_{\mathrm{P}_{2}}= & -2.698-1.38 x \\
& -2 \log _{10}((0-x) / x), \quad 1773 \mathrm{~K} \\
\log _{10} p_{\mathrm{P}_{2}=}= & -3.052-1.25 x \\
& -2 \log _{10}((0-x) / x), \quad 1673 \mathrm{~K} \\
\log _{10} p_{\mathrm{P}_{2}=}= & -3.610-0.95 x \\
& -2 \log _{10}((\theta-x) / x), \quad 1573 \mathrm{~K} \\
\log _{10} p_{\mathrm{P}_{2}=} & -4.292-0.87 x \\
& -2 \log _{10}((0-x) / x), \quad 1473 \mathrm{~K}
\end{aligned}
$$

For $\mathrm{FeP}_{x}$, the re-construction of $p_{\mathrm{P}_{2}}-\mathcal{T}-x$ relationships may be made with $\theta=0.5$. The results are summarized in Fig. 5, together with the experimental results $^{1)}$ for the sake of comparison. The agreement between the estimated and the experimental values appears to be reasonable.

Figure 6 shows the results for estimation of the $p_{\mathrm{P}_{2}}-x$ relationships for $\mathrm{FeC}_{y} \mathrm{P}_{x}$ at $1673 \mathrm{~K}$ with the choice of $\theta$-values based on the results given in Fig. 4; i.e.,

$$
0\left(\mathrm{FeC}_{y} \mathrm{P}_{x}\right)=0.5-1.25 y
$$

The experimental result $\mathrm{s}^{2)}$ appear to be in reasonable accord with the estimation; for example, points 6 ( $y=$ $0.052)$ and $7(y=0.051)$ are on the estimated curve for $\mathrm{FeC}_{0.05} \mathrm{P}_{x}$.

\section{Comparison with the Thermodynamic Analysis}

Ban-ya et al. derived thermodynamically the value of $-117.2 \mathrm{~kJ} \mathrm{~mol}^{-1}$ for the enthalpy $\Delta H^{\theta}$ of $\mathrm{P}$ solution in molten Fe ( $c f$. , Eq. (40) in Ref. 1)). On the other hand, the following expression may be given for the enthalpy $\Delta H$ in terms of the parameters appearing in the present analysis:

$$
\Delta H=6 E_{\mathrm{P}-\mathrm{Fe}}+12 x E_{\mathrm{P}-\mathrm{P}}+D_{\mathrm{P}_{2}} / 2
$$

Value of $6 E_{\mathrm{P}-\mathrm{re}}$ is given by Eq. (6) and the value of $D_{\mathrm{P}_{2}}$ is about $248 \mathrm{~kJ} \mathrm{~mol}^{-1}{ }^{13}$ ) Thus, the contribution of the sum $\left(6 E_{\mathrm{P}-\mathrm{re}}+D_{\mathrm{P}_{2}} / 2\right)$ to $\Delta H$ is estimated to be about $-100 \mathrm{~kJ} \mathrm{~mol}^{-1}$. The order of magnitude of $E_{\mathrm{P}-\mathrm{P}}$ is rather small; e.g., the term, $12 x E_{\mathrm{P}-\mathrm{P}}$, would give a contribution of no more than $-5 \mathrm{~kJ} \mathrm{~mol}^{-1}$ to $\Delta H$ at the composition of $x=0.2$. Thus, the discrepancy between $\Delta H^{\theta}$ estimated by Ban-ya et al. ${ }^{1)}$ and 


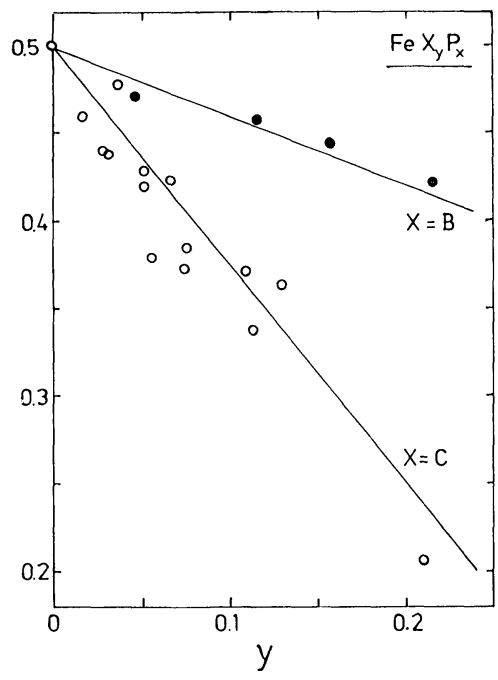

Fig. 4. Estimated values of $\theta$ for molten $\mathrm{FeX}_{y} \mathrm{P}_{x}$ as a function of $y(\mathrm{X}=\mathrm{B}$, C).

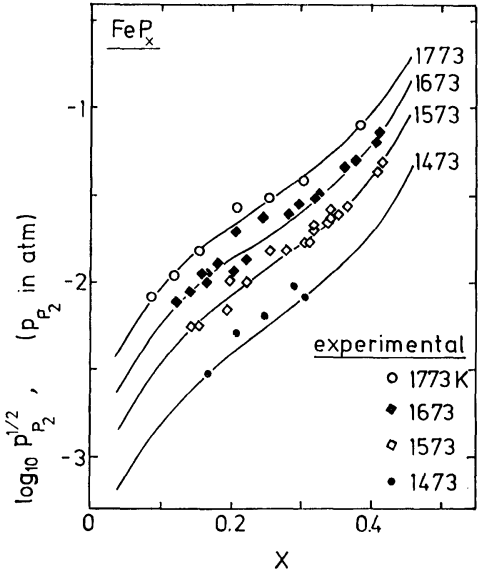

Fig. 5. Estimated $\log _{10} p_{\mathbf{P}_{2}}^{1 / 2} v s . x$ relationships for molten $\mathrm{FeP}_{x}$ compared with the experimental data. ${ }^{11}$

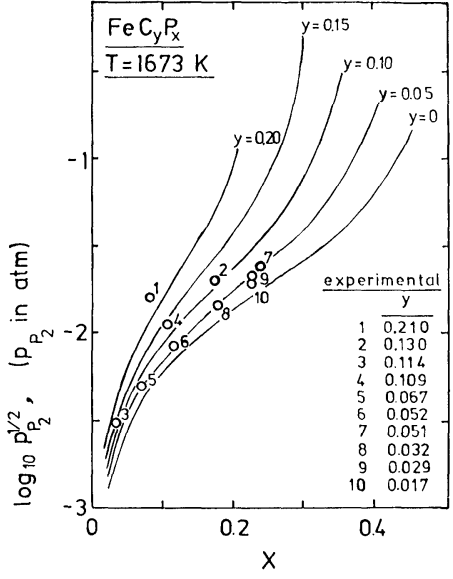

Fig. 6. Estimated $\log _{10} p_{1_{2}}^{1 / 2} v s$. $x$ relationships for molten $\mathrm{FeC}_{y} \mathrm{P}_{x}$ compared with the experimental data. ${ }^{2}$
$\Delta H$ estimated in the present work is $c a .10 \mathrm{~kJ} \mathrm{~mol}^{-\mathbf{1}}$. This discrepancy does not appear to be very serious, considering that, in the present analysis, by taking $0=0.5$, the experimental $p_{\mathrm{P}_{2}}-T-x$ data from all the composition range $(x<0.42)$ reported by Ban-ya et al. ${ }^{1)}$ were used, while in their own analysis, the data for $x>1 / 3$ were discarded.

One of the advantages of statistical thermodynamics relative to the conventional thermodynamics is that all the parameters appearing in the equations have clear physical meanings with the reference state of energy zero being taken at the infinite separation of each component atom at rest, while thermodynamics usually gives the relative values, which are entirely dependent on the choice of arbitrary reference state.

\section{Summary}

Experimental $p_{\mathrm{P}_{2}}-T-x$ relationships reported by Ban-ya et al. for molten $\mathrm{FeP}_{x}{ }^{1)}$ and molten $\mathrm{FeX}_{y} \mathrm{P}_{x}$ $\left.(\mathrm{X}=\mathrm{B}, \mathrm{G})^{2}\right)$ were analysed by statistical thermodynamics on the basis of a priori assumption of constant $E_{\mathrm{P}-\mathrm{P}}$ at a given temperature. This a priori assumption may be met by properly choosing the parameter 0 . While the $p_{P_{2}}-T-x$ data for the composition range of $x>1 / 3$ were not involved for the thermodynamic analysis of $\mathrm{FeP}_{x},{ }^{1)}$ the experimental data for all the composition range $(x<0.42)$ were used in the present statistical thermodynamic analysis.

The value of $E_{\mathrm{P}-\mathrm{re}}$ was estimated to be about -58 $\mathrm{kJ} \mathrm{mol}^{-1}(c f$. ., Eq. (6)). Interaction between $\mathrm{P}$ atoms in molten Fe was estimated to be weak and attractive (varying from $\sim-1 \mathrm{~kJ} \mathrm{~mol}^{-1}$ at $1473 \mathrm{~K}$ to $\sim-2$ $\mathrm{kJ} \mathrm{mol}^{-1}$ at $1773 \mathrm{~K}$ ).

The following generalized formula may be used for the estimation of the $p_{\mathrm{P}_{2}}-T-x$ relationships for $\mathrm{FeP}_{x}$ and $\mathrm{FeX}_{y} \mathrm{P}_{x}$ (cf., Eqs. (15) (18)):

$$
\begin{aligned}
\log _{10} p_{\mathrm{P}_{2}}= & (-12.080+0.00534 T) \\
& +(1.86-0.00183 \mathcal{T}) x \\
& -2 \log _{10}((\theta-x) / x) \quad \ldots
\end{aligned}
$$

The value of $\theta$ for $\mathrm{FeP}_{x}$ may be taken as 0.5 . The value of $\theta$ for $\mathrm{FeX}_{y} \mathrm{P}_{x}$ may be given by

$$
0\left(\mathrm{FeX}_{y} \mathrm{P}_{x}\right)=0.5-s y
$$

where, $s$ refers to the extent of blockage of the interstices per fraction of addition of interstitial element $\mathrm{X}$ (e.g., $s=0.4$ for B and $s=1.25$ for $\mathrm{C}(c f .$, Fig. 4$)$ ). If the element $\mathrm{X}$ alters significantly the values of $E_{\mathrm{P}-\mathrm{P}}$, $E_{\mathrm{P}-\mathrm{ro}}$ and $f_{\mathrm{P}}$ in $\mathrm{FeX}_{y} \mathrm{P}_{x}$ from those in $\mathrm{FeP}_{x}$, this approximation would not be valid.

\section{REFERENGES}

1) S. Ban-ya, N. Maruyama and S. Fujino: Tetsu-to-Hagané, 68 (1982), 269.

2) S. Ban-ya, N. Maruyama and S. Fujino: Tetsu-to-Hagané, 69 (1983), 921.

3) N. Shohoji, M. Katsura and T. Sano: J. Less-Common Met., 38 (1974), 59.

4) N. Shohoji: J. Less-Common Met., 90 (1983), L27.

5) N. Shohoji: J. Less-Common Met., 102 (1984), 53.

6) N. Shohoji: J. Less-Common Met., 114 (1986), 249.

7) N. Shohoji: Mater. Lett., 3 (1985), 206.

8) N. Shohoji: Phys. Stat. Sol. (B), 118 (1983), 811.

9) N. Shohoji: Phys. Stat. Sol. (B), 119 (1983), K87.

10) N. Shohoji: Z. Metallkde., 76 (1985), 192.

11) N. Shohoji: J. Nucl. Mater., 127 (1985), 88.

12) N. Shohoji: Mater. Chem. Phys., 13 (1985), 567.

13) JANAF Thermochemical Tables, D. R. Stull. H. Prophet and G. C. Sinke, ed., Dow Chemical Co., Midland MI, (1965).

14) M. Hoch: Trans. TMS-AIME, 230 (1964), 138.

15) M. Hoch: Phase Stability in Metals and Alloys, McGrawHill, New York, (1966), 419.

16) M. Hoch: Anisotropy in Single-Crystal Refractory Compounds, I, Plenum, New York, (1968), 163.

17) J. Chipman: Trans. TMS-AIME, 239 (1967), 1332.

18) S. Ban-ya and J. Chipman: Trans. TMS-AIME, 242 (1968), 940.

19) J. Ghipman: Met. Trans., 3 (1972), 879.

20) K. Schwerdtfeger: Trans. TMS-AIME, 239 (1967), 1432.

21) N. Shohoji: Z. Phys. Chem., Neue Folge (a special volume to be published in 1986 as a proceedings of the Int'l Symp. on Hydrogen in Metals, held in Belfast, March, 1985). 